\title{
Conductance-based Adaptive Exponential integrate-and-fire model
}

\author{
Damien Depannemaecker ${ }^{1^{*}}$, Tomasz Górski $^{1^{*}}$, and Alain Destexhe ${ }^{1}$ \\ ${ }^{1}$ Department of Integrative and Computational Neuroscience, \\ Paris-Saclay Institute of Neuroscience, Centre National de la \\ Recherche Scientifique, France \\ ${ }^{*}$ These authors contributed equally, order chosen randomly.
}

Keywords: point neuron model, integrate-and-fire models, dynamical systems, neural adaptation

\begin{abstract}
The intrinsic electrophysiological properties of single neurons can be described by a broad spectrum of models, from the most realistic HodgkinHuxley type models with numerous detailed mechanisms to the phenomenological models. The Adaptive Exponential integrate-and-fire (AdEx) model has emerged as a convenient "middle-ground" model. With a low computational cost, but keeping biophysical interpretation of the parameters it has been extensively used for simulation of large neural networks. However, because of its current-based adaptation, it can generate unrealistic behaviors. We show the limitations of the AdEx model, and to avoid them, we introduce the Conductance-based Adaptive Exponential integrate-and-fire model (CAdEx). We give an analysis of the dynamic of the CAdEx model and we show the variety of firing patterns that it can produce. We propose the CAdEx model as a richer alternative to perform network simulations with simplified models reproducing neuronal intrinsic properties.
\end{abstract}

\section{Introduction}

Computational modeling of large scale networks requires to compromise between biophysical realism and computational cost. This requirement might be satisfied by many two-variable models (Morris \& Lecar, 1981; Krinskii \& Kokoz, 1973; Fitzhugh, 1961; Izhikevich, 2003; Brette \& Gerstner, 2005). In particular, two-variable models are largely used and studied, the Izhikevich model (Izhikevich, 2003) and the Adaptive Exponential Integrate and Fire (AdEx) model (Brette \& Gerstner, 2005; Naud, Marcille, Clopath, \& Gerstner, 2008). 
The first variable of these models corresponds to membrane voltage, the second, usually slower changing variable, corresponds to neural adaptation and allows achieving more complex dynamics and firing patterns, which can be observed in neural recordings. In both models the second variable has a form of additional current flowing into a neuron, which may lead to unrealistic changes of membrane voltage, especially in the case of long and intense neuronal firings such as observed during seizures (McCormick \& Contreras, 2001). Here, we describe this limitation, and to avoid it, we propose a modification of the second variable dynamics. The adaptation variable in our model has the form of a conductance, introducing the Conductance-based Adaptive Exponential Integrate and Fire model or $C A d E x$ model. Previous work has used conductance-based adaptation, but these models used were either more simplified (Treves, 1993) or much more detailed Hodgkin-Huxley type models with complex channel gating dynamics (Connor \& Stevens, 1971). Our model kept the advantage of the simplified two-variables models, while extending the repertoire of possible subthreshold and superthreshold dynamics.

\section{Conductance-based Adaptive Exponential model}

The Adaptive Exponential integrate and fire model can reproduce many types of firing patterns: tonic spiking cells, regular spiking adaptive cells, bursting cells, etc (Naud et al., 2008; Clopath, Jolivet, Rauch, Lüscher, \& Gerstner, 2007). However, because the adaptation in AdEx model is a current, a strong and unrealistic hyperpolarization of cell can appear after a period of prolonged firing (Fig. 1). Moreover, subthreshold adaptation in this model is linear which means that in the periods of strong hyperpolarization the adaptation current does not deactivate and can remain unrealistically strong. For instance, modelling Mcurrent mediated adaptation in this model poses a problem because M-channels are mostly closed when membrane voltage remains below $-60 \mathrm{mV}$ (Brown \& Adams, 1980). A similar problem happens for strong depolarization due to lack of saturation of the adaptation current.

To overcome these problems, we propose here a model with conductance based adaptation $g_{A}$ and with a sigmoid dependence of subthreshold adaptation on voltage. We named it Conductance based Adaptive Exponential model $C A d E x$. The equations of the model are as follows:

$$
\begin{gathered}
C \frac{d V}{d t}=g_{L}\left(E_{L}-V\right)+g_{L} \Delta_{T} \exp \left(\frac{V-V_{T}}{\Delta_{T}}\right)+g_{A}\left(E_{A}-V\right)+I_{s} \\
\tau_{A} \frac{d g_{A}}{d t}=\frac{\bar{g}_{A}}{1+\exp \left(\frac{V_{A}-V}{\Delta_{A}}\right)}-g_{A}
\end{gathered}
$$

with after-spike reset mechanism:

$$
\text { if } V \geq V_{D} \text { then }\left\{\begin{array}{l}
V \rightarrow V_{R} \\
g_{A} \rightarrow g_{A}+\delta g_{A}
\end{array}\right.
$$


where $C$ is the membrane capacitance, $g_{L}$ and $E_{L}$ are the leak conductance and reversal potential. $V_{T}$ is a spike threshold and $\Delta_{T}$ is a slope of the spike initiation, $E_{A}$ is the reversal potential of the adaptation conductance and $I_{s}$ is an input current. In the second equation, $\tau_{A}$ is the time constant of adaptation, $\bar{g}_{A}$ is the maximal subthreshold adaptation conductance, $V_{A}$ is the subthreshold adaptation activation voltage, and $\Delta_{A}$ is the slope of subthreshold adaptation.

Spike is initiated when $V$ approaches $V_{T}$ and exponential term escalates. After reaching a detection limit $V_{D}$, the voltage is reset to the reset potential $V_{R}$ and it remains at this value during a refractory period $\Delta t_{r e f}$. After each spike, $g_{A}$ is incremented by a quantal conductance $\delta g_{A}$.

The sigmoidal subthreshold adaptation function is always positive, i.e. $\bar{g}_{A} \geq$ 0 , and it can be monotonically increasing, $\Delta_{A}>0$, or decreasing, $\Delta_{A}<0$, function of membrane voltage. In the simulations, the value of the detection limit has been set to $V_{D}=-40 \mathrm{mV}$ and the value of the refractory period to $\Delta t_{\text {ref }}=5 \mathrm{~ms}$.

\section{Dynamical analysis of the model}

\subsection{Fixed points and bifurcations}

The CAdEx is a nonlinear dynamical system with discontinuous post-spike reset mechanism. The $V$ and $g_{A}$ nullclines are as follows (Fig.2):

$$
\begin{aligned}
& g_{A}=g_{L} \frac{E_{L}-V}{V-E_{A}}+\frac{g_{L} \Delta_{T}}{V-E_{A}} \exp \left(\frac{V-V_{T}}{\Delta_{T}}\right)+\frac{I_{s}}{V-E_{A}} \\
& g_{A}=\frac{\bar{g}_{A}}{1+\exp \left(\frac{V_{A}-V}{\Delta_{A}}\right)}
\end{aligned}
$$

The intersections of nullclines give fixed points of the system

$$
I_{s}=-g_{L}\left(E_{L}-V\right)-g_{L} \Delta_{T} \exp \left(\frac{V-V_{T}}{\Delta_{T}}\right)-\frac{\bar{g}_{A}}{1+\exp \left(\frac{V_{A}-V}{\Delta_{A}}\right)}\left(E_{A}-V\right)
$$

The analysis of the above equation, which we can write in a simpler form defining new function $S(V)$ as $I_{s}=S(V)$, gives us the number of fixed points for a given input current Fig.2. Function $\mathrm{S}(\mathrm{V})$ tends to $-\infty$ for $V \rightarrow \pm \infty$ and it can have:

1. One global maximum $V_{\max }$, which corresponds to a maximum of two possible intersections between nullclines. Let $I_{R}=S\left(V_{\max }\right), I_{R}$ is then rheobase current above which the system starts to spike spontaneously. For current input $I_{s}<I_{R}$ there are therefore 2 fixed points $V^{-}\left(I_{s}\right)$ and $V^{+}\left(I_{s}\right)$, For $I_{s}=I_{R}$ there is one fixed point and for $I_{s}>I_{R}$ there are no fixed points. 
AdEx
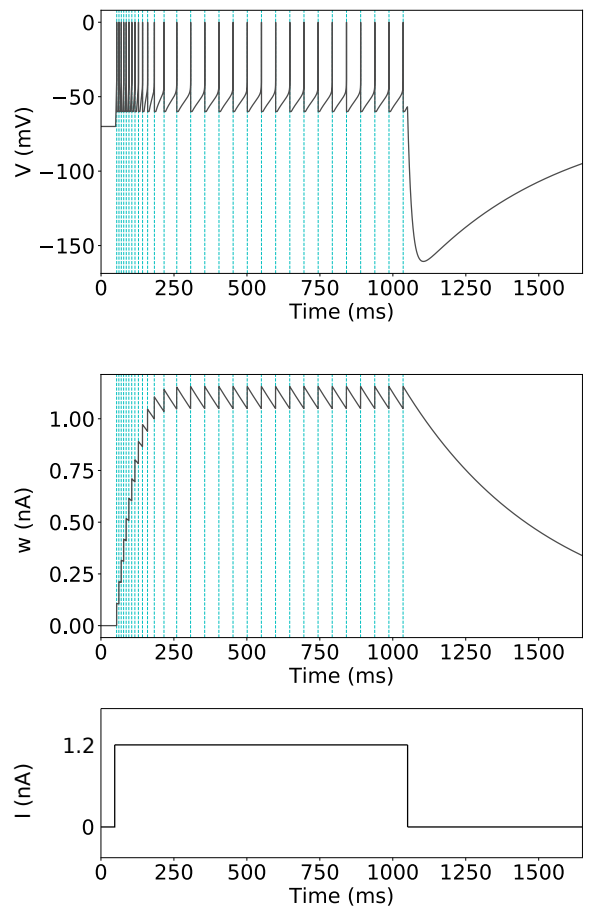

Cadex
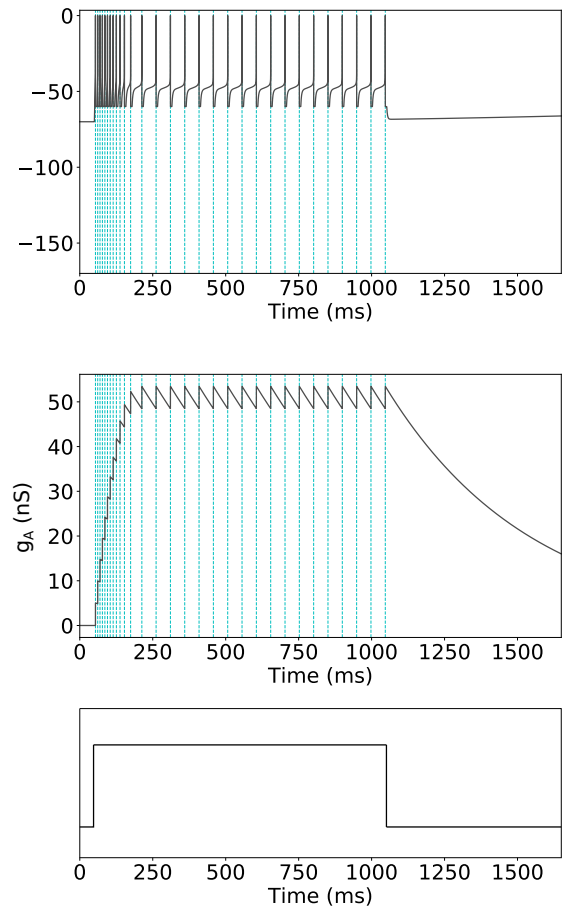

Figure 1: The comparison between AdEx and CAdEx models. Both cells were injected with the same current pulse. Their firing rates $f$ and adaptation parameters $A$ were the same during pulse, $f=30 \mathrm{~Hz}$ and $A=0.03$. However after pulse AdEx cell hyperpolarizes below $-150 \mathrm{mV}$ while CAdEx cell hyporpolarizes to proximity of the adaptation reversal potential, $E_{A}=-70 \mathrm{mV}$. The mathematical definition of the adaptation parameter, $A$, is given in Section 7 . 
2. Two local maxima $V_{\text {max }}^{1,2}$, and one local minimum $V_{\text {min }}$, which corresponds to maximally four possible fixed points. For input current $I_{s}<S\left(V_{\min }\right)$ there are two fixed points, one stable and one unstable. For $S\left(V_{\min }\right)<$ $I_{s}<\min \left\{S\left(V_{\max }^{1,2}\right)\right\}$ there are four fixed points, from which two can be stable. For $\min \left\{S\left(V_{\max }^{1,2}\right)\right\}<I_{s}<\max \left\{S\left(V_{\text {max }}^{1,2}\right)\right\}$ there are two fixed points, and for $I_{s}>\max \left\{S\left(V_{\max }^{1,2}\right)\right\}$ there are no fixed points and neuron fires spontaneously.

To analyze the bifurcations of the system we need to consider the behavior of Jacobian matrix at fixed points $i$ as a function of the input current, $L_{i}\left(I_{s}\right)$, see Appendix.

The trajectories in the phase space of trace $\tau\left(I_{s}\right)=\operatorname{tr}\left(L_{i}\left(I_{s}\right)\right)$ and determinant $\Delta_{i}\left(I_{s}\right)=\operatorname{det}\left(L_{i}\left(I_{s}\right)\right)$ of Jacobian give us the type of bifurcation, Fig.3.

In the case (1), i.e. when there are maximally two possible fixed points, the stable fixed point undergoes Saddle-Node bifurcation if for rheobase current $I_{R}$

$$
\frac{1}{\tau_{A}}>-\frac{\bar{g}_{A}\left(E_{A}-V^{-}\left(I_{R}\right)\right)}{C \Delta_{A}} \frac{\exp \left(\frac{V_{A}-V^{-}\left(I_{R}\right)}{\Delta_{A}}\right)}{\left(1+\exp \left(\frac{V_{A}-V^{-}\left(I_{R}\right)}{\Delta_{A}}\right)\right)^{2}}
$$

Otherwise the bifurcation is of Andronov-Hopf type, see Fig.3. Since $E_{A}<$ $V^{-}\left(I_{R}\right)$, then if $\Delta_{A}<0$ bifurcation is always of Saddle-Node type. If $\Delta_{A}>0$, both types are possible depending on the condition (5).

\subsection{Subthreshold oscillations}

The system can oscillate around equilibrium if (a) the equilibrium is stable, i.e. $\tau\left(I_{s}\right)<0$ and $\Delta\left(I_{s}\right)>0$ and (b) eigenvalues of Jacobian at fixed point have imaginary part, i.e. $\tau^{2}-4 \Delta<0$. In that case, the frequency of oscillations is given by $\nu=\frac{1}{4 \pi} \sqrt{4 \Delta-\tau^{2}}$, see Fig. 4 and Appendix.

Sustained subthreshold oscillations due to the emergence of a limit cycle can be also observed. Some examples in the case of multi-stability are given in the next section.

\section{Multi-stability}

In this section, we present a list of examples of multi-stability. Such behaviors can be observed due to two properties of the model.

Subthreshold multi-stability. Due to the non-linear form of the subthreshold adaptation, the system may exhibit two or four fixed points. The case of two fixed points has been described in the previous section. With four fixed points, various configurations are possible: with a positive or negative slope $\left(\Delta_{A}\right)$ of the adaptation nullcline. The stability of these fixed points, depending on the input current, are shown in Fig.2.

Superthreshold stability. The model has a discontinuity which occur after spike and corresponds to the reset of membrane voltage and to the increment of 
a

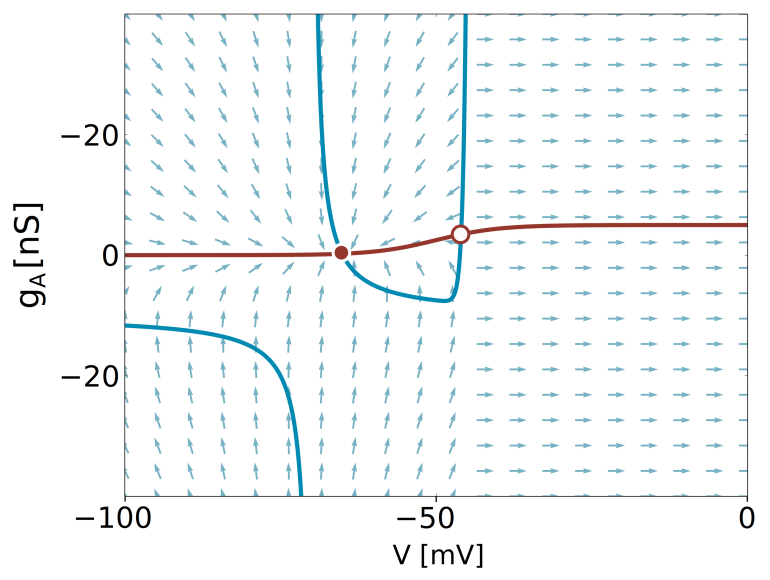

Nullclines of CAdEx system

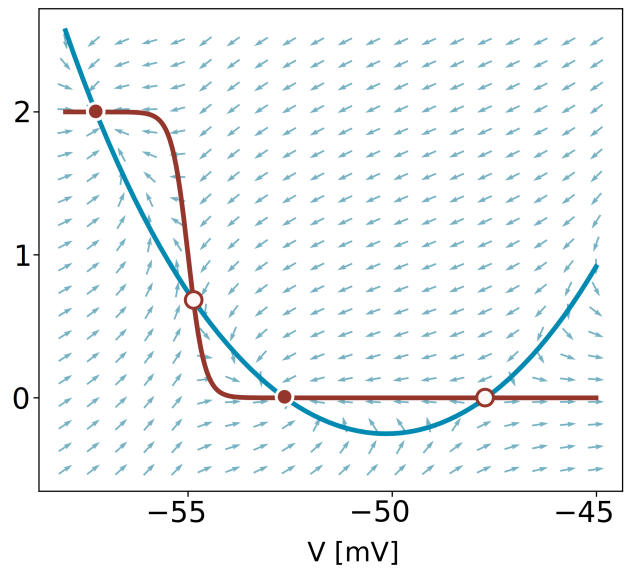

Input current and fixed points

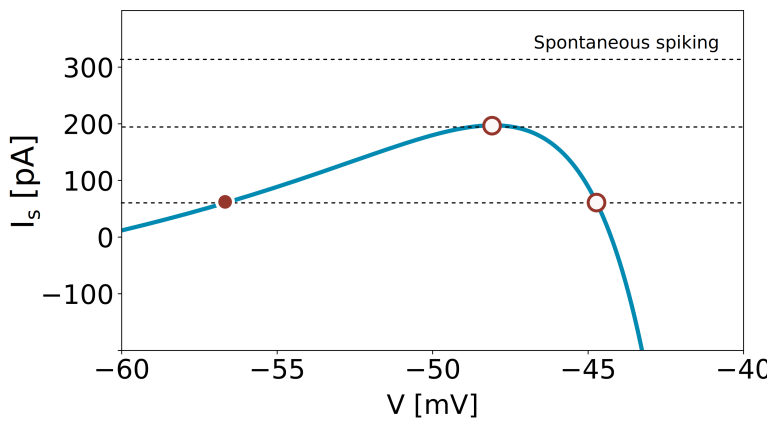

Figure 2: (a) Nullclines of CAdEx system for two parametrizations, left for $\Delta_{A}>0$, right for $\Delta_{A}<0$. The blue line is a V-nullcline, red line - $g_{A^{-}}$ nullcline. The red filled circle indicates stable fixed point, white the filled circle - unstable fixed point. Arrows indicate the direction of the vector field. Increase of input current moves the V-nullcline up. (b) Corresponding $S(V)$ functions. Intersections with lines of constant input current $I_{s}$ indicate positions of the fixed points. 


\section{a} Poincaré diagram

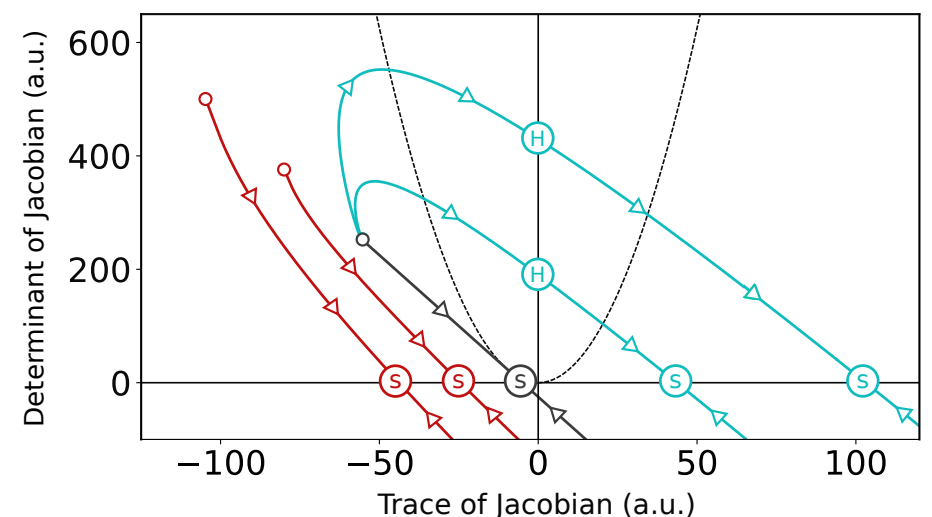

(S) Saddle-Node

(H) Andronov-Hopf

$\triangleright$ Increase of input current

- $\Delta_{\mathrm{A}}$ positive

- $\Delta_{\mathrm{A}}$ negative

- No subthreshold adaptation
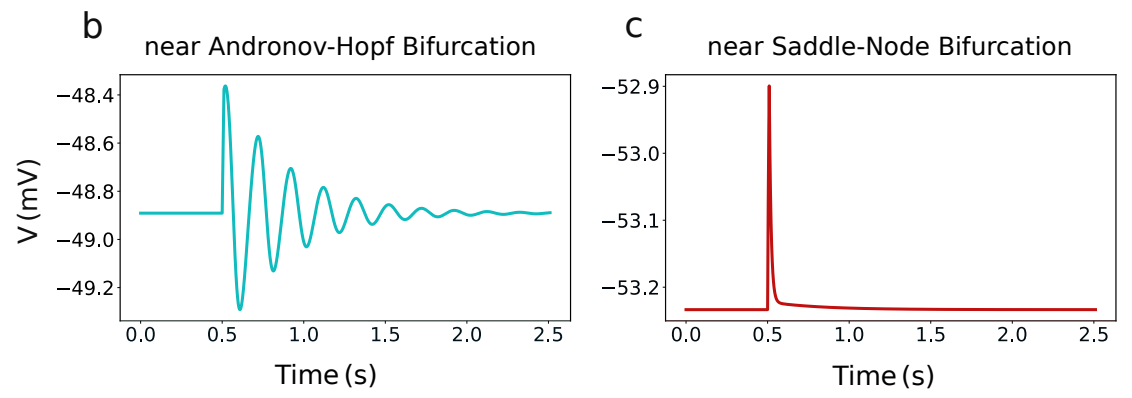

Figure 3: Bifurcations of the CAdEx system. (a) Trajectories of the fixed points on the Poincaré diagram for increasing input current $I_{s}$. For positive $\Delta_{A}$, blue lines, the stable fixed point loses stability with an Andronov-Hopf bifurcation, $\bar{g}_{A}=10$ and $20 \mathrm{nS}$. For negative $\Delta_{A}$, red lines, the stable fixed point merges with the saddle point in a Saddle-Node bifuraction, $\bar{g}_{A}=5$ and $10 \mathrm{nS}$. Without subthreshold adaptation, $\bar{g}_{A}=0 \mathrm{nS}$, black line, the system undergoes a SaddleNode bifurcation. (b, c) Response of the cell to a brief current pulse, (b) near the Andronov-Hopf bifurcation, (c) near the Saddle-Node bifurcation. 

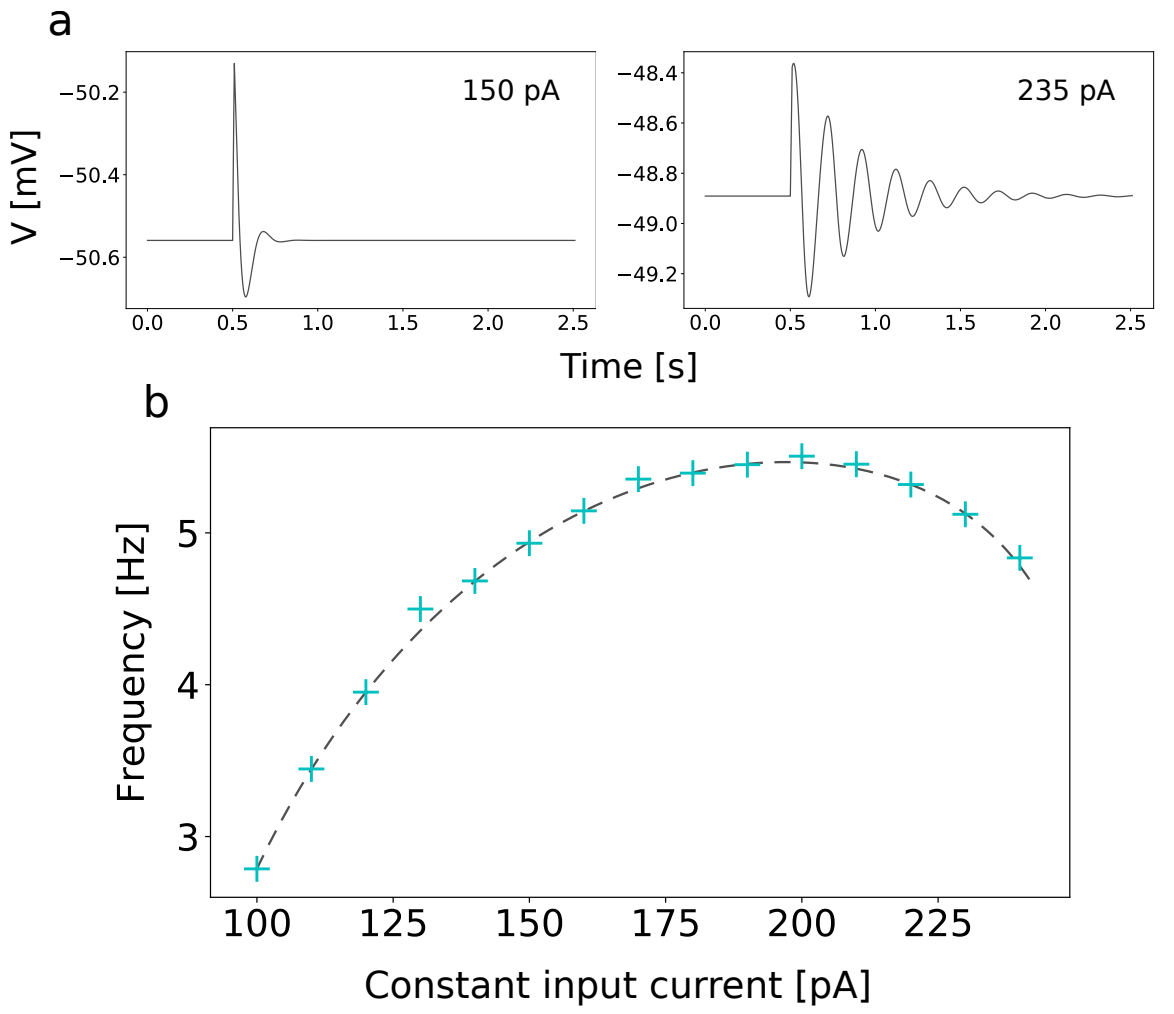

Figure 4: (a) Subthreshold oscillations of the membrane voltage around the stable equilibrium for different constant input currents. (b) Frequency of subthreshold membrane voltage oscillations in the CAdEx model as a function of a constant input current. Crosses signify numerical results, dashed line theoretical prediction, $\nu=\frac{1}{4 \pi} \sqrt{4 \Delta-\tau^{2}}$. The membrane voltage was perturbed from the stable equilibrium for a given constant input current, by a short (10 ms) pulse of added current (10 pA). 
adaptation conductance. It can lead to steady-state firing behavior, which can be treated as an attractor of the model. The reset discontinuity has, in many aspects, the same effect as a third variable. It allows chaotic behaviors (as described in the next section) normally impossible in a continuous two dimensional system.

Here, we give three examples of multi-stability: (a) with four fixed points and negative slope of adaptation, (b) with four fixed points and positive slope of adaptation and (c) with two fixed points and positive slope of adaptation.

(a) With a negative and small enough value of $\Delta_{A}$, the nullclines can have four crossings. In this case, the system has two stable fixed points with different values of the membrane potential. In this situation, three stable steady-states are observed, Fig.5a: Two possible resting state of membrane potential and a self-sustained spiking. Small perturbations permit switch between these steady-states, as shown in Fig. 5b. By applying synaptic noise through conductance-based inhibitory and excitatory synapses (see Appendix), oscillations between these stable states can be observed, Fig. $5 \mathrm{c}$.

(b) With a positive and small enough value of $\Delta_{A}$, the system can also have four fixed points. In this case, the emergence of a stable limit cycle is observed. It leads to three possible stable behavior: resting state, subthreshold oscillations, and regular spiking as show in Fig.6a. As in the previous condition, oscillations between stable states can be observed under synaptic bombardment, Fig.6b.

(c) In a situation with two fixed points, the emergence of an unstable limit cycle is observed, leading to bi-stability near the threshold between spiking and, non-spiking damped oscillations, as shown in Fig.7c.

\section{$5 \quad$ Firing patterns}

The CAdEx model is able to reproduce a large repertoire of intrinsic electrophysiological firing patterns. In this section, the dynamics of chosen firing patterns in CAdEx will be detailed. The initialization values of the two variables in simulation were $V(0)=-60 \mathrm{mV}$ for the membrane potential and $g_{A}(0)=0 \mathrm{nS}$ for the adaptation; except when $\Delta_{A}<0$ when $g_{A}$ is initialized at $g_{A}^{\infty}(-60 \mathrm{mV})$. For sets of parameters used in this section, the system does not have fixed points. Therefore, independently of the starting point location, the system converges to the same steady state firing. However behaviors in the transient regime may change, which is crucial for spike frequency adaptation. Parameters for each pattern of Fig. 8 are in Table 1.

Adaptive spiking. The adaptation can cause a decrease of spiking frequency as seen in Fig. 8a. Correspondingly the value of $g_{A}$ increases until it reaches a steady state. The spike frequency adaptation is mainly affected by spike 
a.
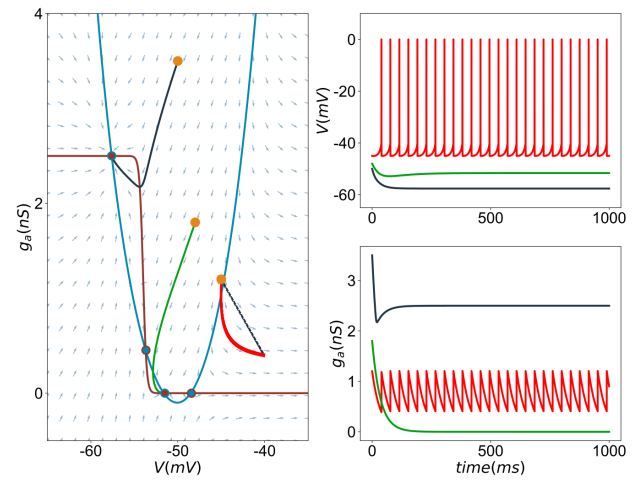

b.
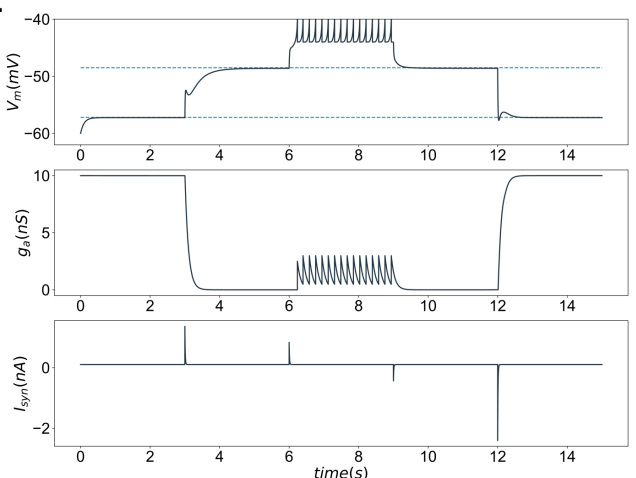

C.
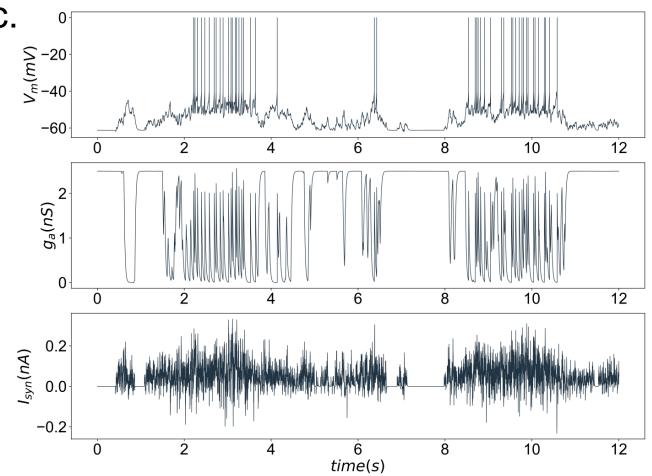

Figure 5: Multi-stability observed with a negative slope of $g_{a}$ : (a) the dynamics for single neuron with two stable fixed-points and a stable limit cycle due to the reset, (b) transitions between possible steady-states due to excitatory or inhibitory short-time perturbations, (c) transition between up and down state of single neuron receiving input through conductance-based synapses. 
a.

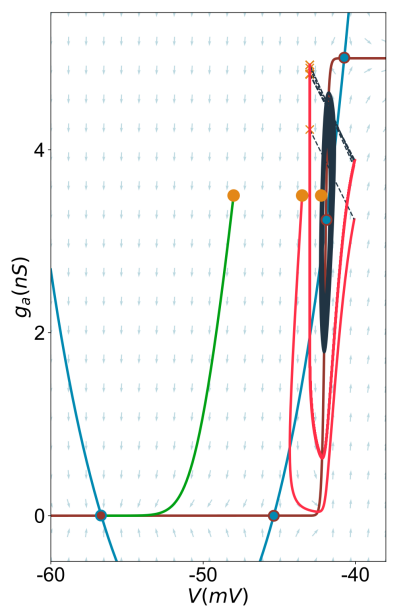

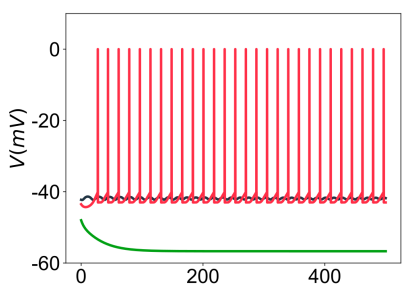

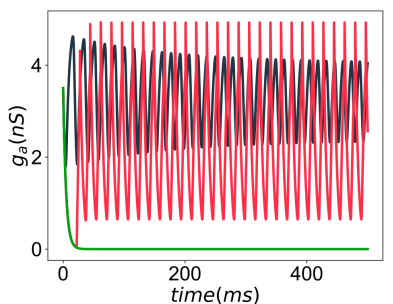

b.
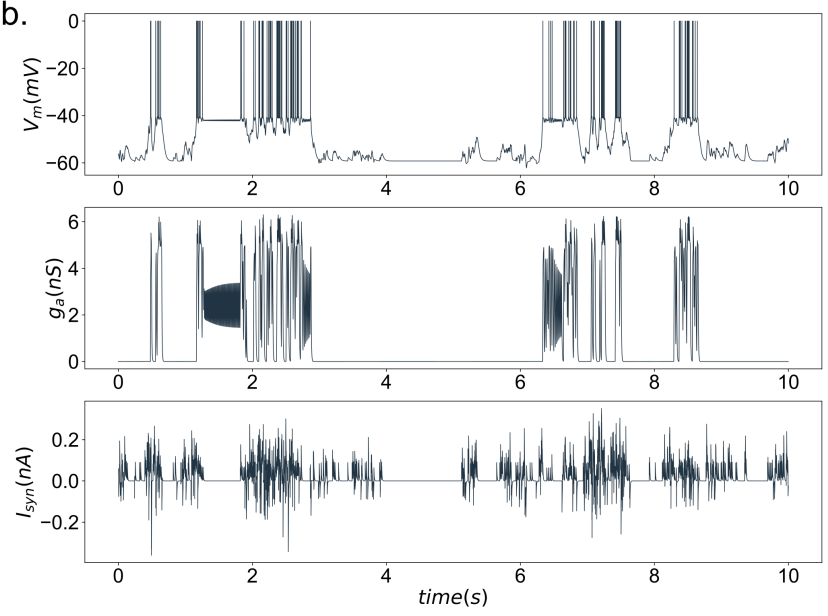

Figure 6: Multi-stability observed with a positive slope of $g_{a}$ : (a) the dynamics for single neuron with a stable fixed-point: a stable limit cycle due to the subthreshold dynamics and a limit cycle due to the reset, (b) transitions between up and down state of single neuron receiving input through conductance-based synapses. 



Figure 7: Multi-stability observed with two fixed points, (a) damped subthreshold oscillations are observed near the spiking behavior. (b) Enlarged concerned region (blue square). Two simulations initiated: inside (red) and outside (dark grey) the unstable limit cycle 
triggering parameters $\left(V_{T}\right.$ and $\left.\Delta_{T}\right)$, membrane properties: $C$ and $g_{L}$, as well as the adaptation parameters, $g_{A}$ and strongly by $\Delta g_{A}$.

Tonic spiking. In the absence of adaptation, i.e. $\bar{g}_{A}=0$, the model exhibits tonic spiking. However, tonic spiking can also be observed with non-zero adaptation, as in Fig. 8b, if the steady state firing can be reached after the first spike. The steady state spiking frequency is be influenced by membrane capacitance, $C$, the leak conductance, $g_{L}$, and the slope of spike initiation $\Delta_{T}$, and also by the adaptation time constant $\tau_{A}$. The hyperpolarization rebound after spiking depends on both: reset value $V_{R}$ and adaptation time constant $\tau_{A}$.

Bursting. To obtain bursting behaviors, Fig.8c, the reset value, $V_{R}$, has to be higher than the voltage of the minimum of the $V$ nullcline. The system spikes until it crosses the $V$ nullcline, ending the burst. Then, above the $V$ nullcline, the system is driven to lower values of adaptation and membrane potential. After a second crossing of the $V$ nullcline, the system spikes again, starting a new bursting cycle. Bursts can be characterized by intra- and inter-bursts activities. The inter-burst activity is affected by adaptation, $\bar{g}_{A}$ and $\tau_{A}$ strongly determine the inter-burst time interval and after burst hyperpolarization.

Delayed spiking. All firing patterns can occur with a time delay. Example is given for bursting, Fig. 8d, and low frequency spiking Fig.8b. The distance between $V$ and $g_{A}$ nullclines which is determined by $I_{s}$. By changing this distance and the time constant $\tau_{A}$ it is possible to obtain a region of slow flow (small $\frac{d V}{d t}$ ) and so increase the delay and the inter-event interval. The reset $V_{R}$ and $\delta g_{A}$ also affects the time required for the system to go around the $V$ nullcline, and then, to the next event.

Accelerated spiking. A negative slope of $g_{A}\left(\Delta_{A}<0\right)$ associated with $\delta g_{A}=$ 0 , leads to firing rate acceleration as shown in Fig8e.

Chaotic-like spiking. As the CAdEx model is not continuous due to the after spike reset of voltage and incrementation of adaptation, chaotic-like spiking behavior can be observed as shown in Fig.8f. These phenomena occur when the reset is close to the right branch of the $V$ nullcline. To verify that this irregularity is not due to numerical error, we used various integration methods and various time steps in Brian2 simulator (Stimberg, Brette, \& Goodman, 2019).

\section{Adaptation and irregularity}

A convenient way to measure spike frequency adaptation is given by the adaptation index:

$$
A=\frac{1}{N-1} \sum_{i=1}^{N-1} \frac{\mathrm{ISI}_{i+1}-\mathrm{ISI}_{i}}{\mathrm{ISI}_{i+1}+\mathrm{ISI}_{i}}
$$

where $N$ is the number of subsequent interspike intervals, ISI $_{i}$. Note that $A \in$ $(-1,1)$ and is positive for decelerating spike trains and negative for accelerating spike trains. 
a.
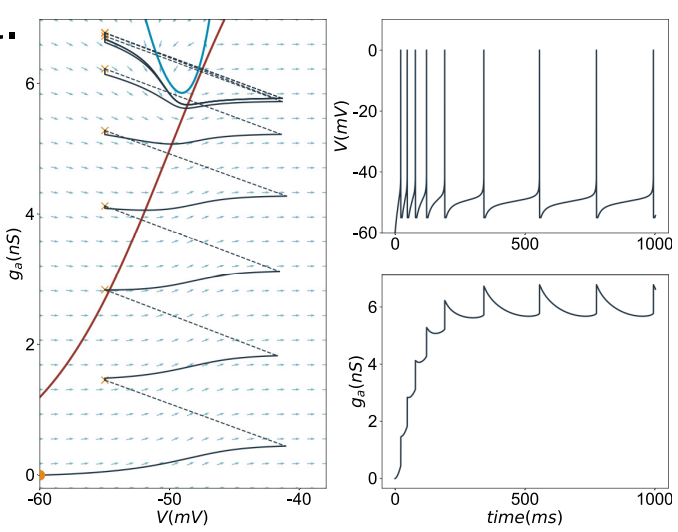

C.
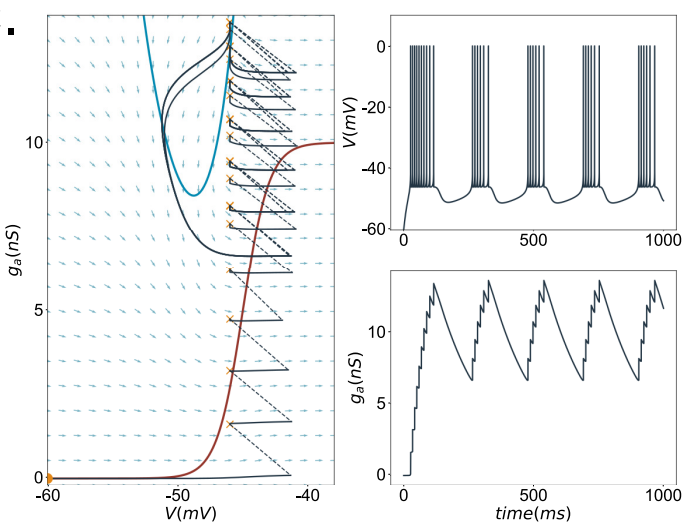

e.
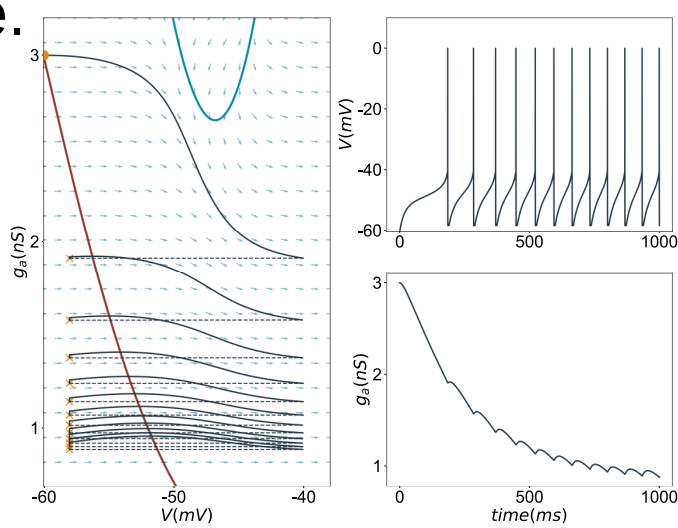

b.
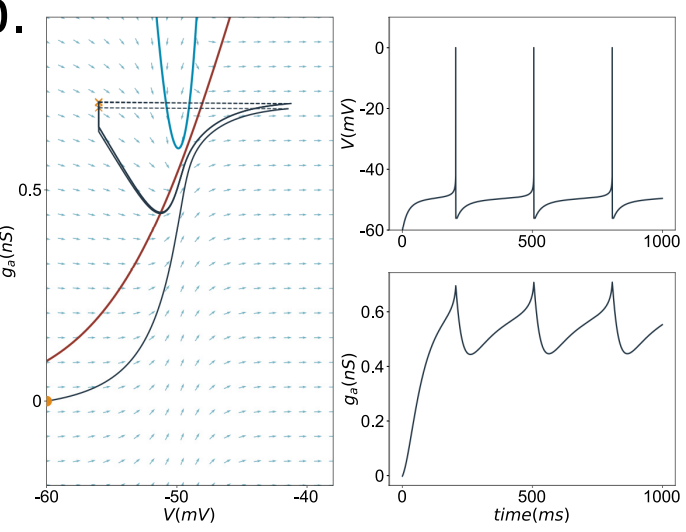

d.
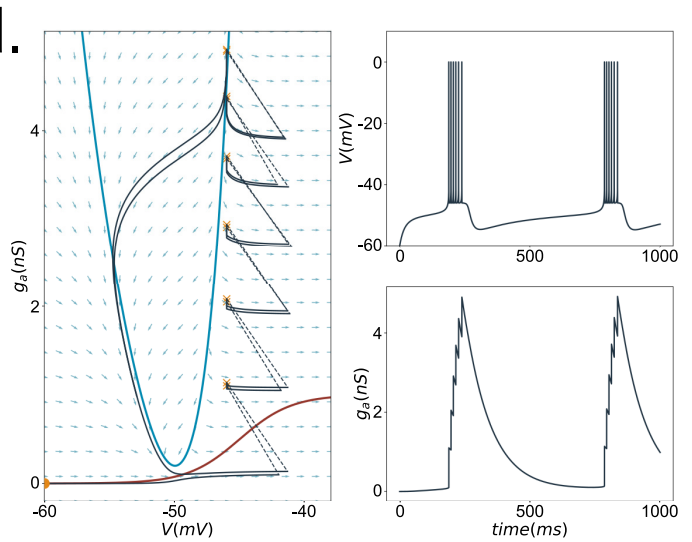

f.
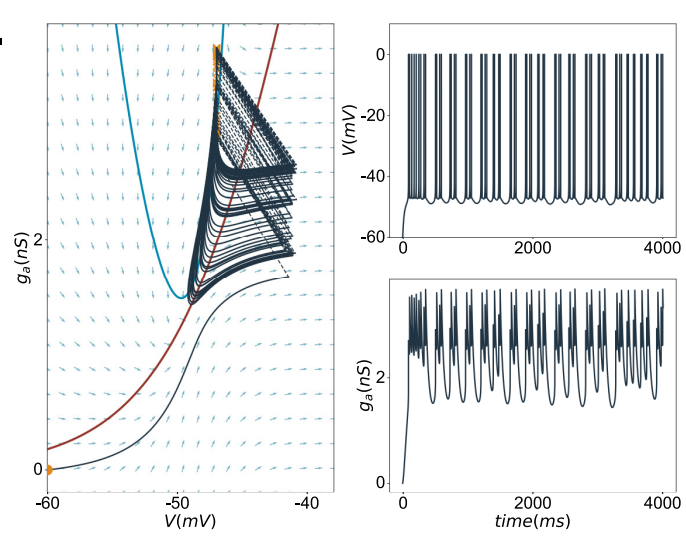

Figure 8: Firing patterns of the CAdEx model: (a) adaptation, (b) delay lowfrequency tonic spiking, (c) burst, (d) delayed burst, (e) acceleration, (f) irregular chaotic-like 


$\begin{array}{lllllll} & C_{m}[\mathrm{pF}] & E_{A}[\mathrm{mV}] & E_{L}[\mathrm{mV}] & I_{s}[\mathrm{pA}] & V_{A}[\mathrm{mV}] & \Delta_{A}[\mathrm{mV}] \\ \text { Adaptive spiking } & 200 & -70 & -60 & 200 & -50 & 5 \\ \text { Tonic spiking } & 200 & -70 & -70 & 192 & -45 & 5 \\ \text { Bursting } & 200 & -60 & -58 & 150 & -45 & 1 \\ \text { Delayed burst } & 200 & -70 & -60 & 100 & -45 & 2 \\ \text { Accelerated spiking } & 200 & -70 & -60 & 130 & -60 & -5 \\ \text { Chaotic-like spiking } & 200 & -70 & -58 & 90 & -40 & 5 \\ & & & & & & \\ & V_{R}[\mathrm{mV}] & V_{T}[\mathrm{mV}] & \delta g_{A}[\mathrm{nS}] & \bar{g}_{A}[\mathrm{nS}] & g_{L}[\mathrm{nS}] & \tau_{A}[\mathrm{~ms}] \\ \text { Adaptive spiking } & -55 & -50 & 1 & 10 & 10 & 200 \\ \text { Tonic spiking } & -56 & -50 & 0 & 2 & 10 & 40 \\ \text { Bursting } & -46 & -50 & 1 & 10 & 10 & 200 \\ \text { Delayed burst } & -46 & -50 & 1 & 1 & 12 & 100 \\ \text { Accelerated spiking } & -58 & -48 & 0 & 6 & 10 & 300 \\ \text { Chaotic-like spiking } & -47 & -50 & 1 & 10 & 10 & 25\end{array}$

Table 1: The parameters of CAdEx model for firing patterns shown in Fig.8

To measure irregularity of spiking we used the coefficient of variance of ISI:

$$
\operatorname{Ir}=\frac{\sigma_{\text {ISI }}}{\langle\mathrm{ISI}\rangle}
$$

where $\langle$ ISI $\rangle$ and $\sigma_{\text {ISI }}$ are the mean and the standard deviation of ISIs respectively. According to this definition, $\operatorname{Ir} \in[0, \infty)$, where $I r=0$ for regular tonic spiking.

Both subthreshold $\bar{g}_{A}$ and post-spike $\delta g_{A}$ adaptation parameters affect adaptation index, see Fig.9a. This allows the model to reproduce wide range of $A$ index values observed in neurons.

The irregular spiking (like chaotic-like spiking and bursting) is especially pronounced in the transition zone between slow and fast regular spiking regions, see Fig.9b. On the phase diagram slow regular spiking corresponds to postspike reset occurring on the left side of the right branch of the V-nullcline, and, consequently, leading to longer interspike intervals, while fast tonic spiking corresponds to reset occurring on the right side, leading to fast subsequent spike. In the transition zone alternations between resets on the left and right side of $\mathrm{V}$ nullcline can lead to highly irregular spiking (cf.chaotic-like Fig.8f) and bursting Fig.8c and d).

\section{Discussion}

In this paper, we proposed a new integrate-and-fire model with two variables, and which can produce a large repertoire of electrophysiological patterns while still allowing for clear mathematical insights and for large scale simulations. This CAdEx model is completely specified with twelve biophysical parameters, and reproduces qualitatively similar pattern as the AdEx model (Naud et al., 2008), because the $g_{A}$ nullcline may be considered as locally linear and approximates 
(a)

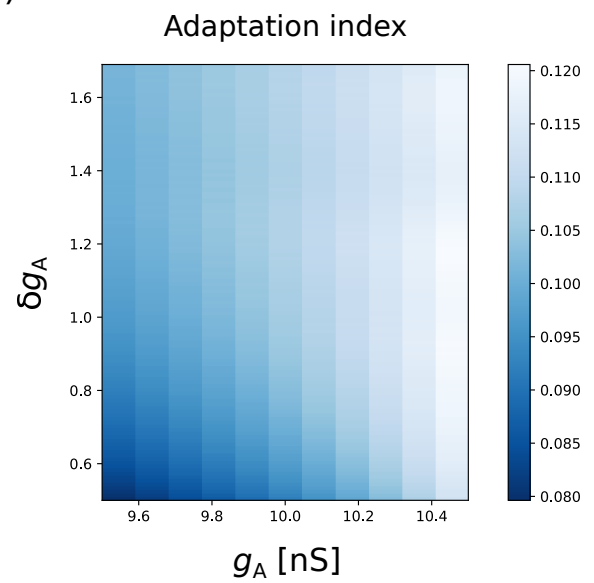

(b)

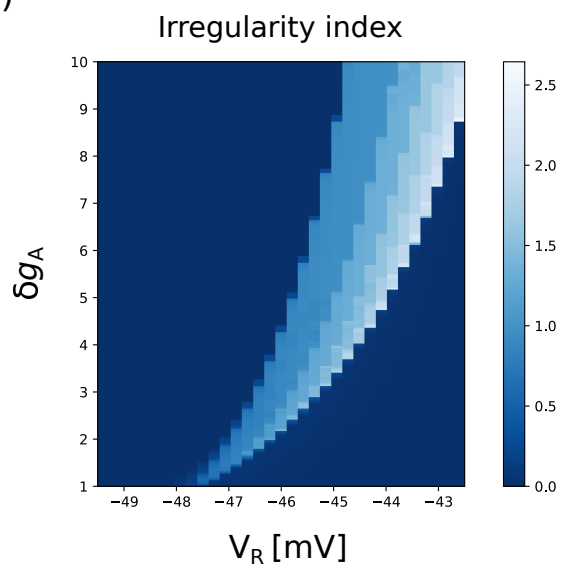

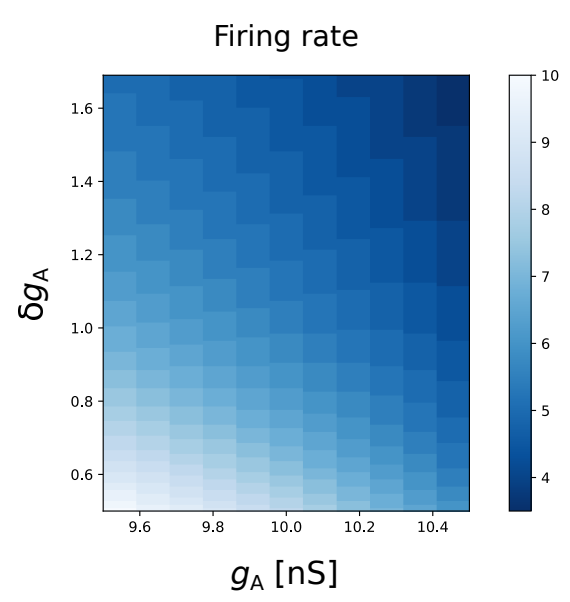

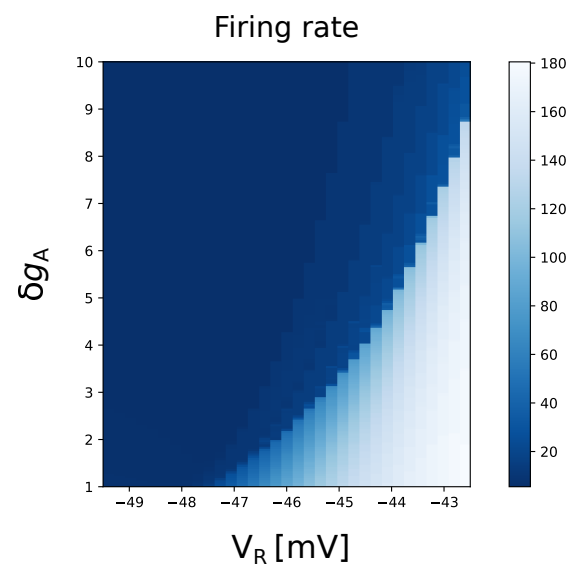

Figure 9: Spike frequency adaptation and spiking irregularity in CAdEx model. (a) Adaptation index $A$ and corresponding firing rate as a function of maximal subthreshold adaptation $\bar{g}_{A}$ and post-spike adaptation $\delta g_{A}$. (b) Irregularity index $I r$ and corresponding firing rate as function of reset potential $V_{R}$ and post-spike adaptation $\delta g_{A}$. 
that of the AdEx model. While the dynamics of the CAdEx model is comparable to the AdEx model for moderate input and firing, the CAdEx model does not suffer from un-naturally strong hyperpolarization after prolonged periods of strong firing. This can be very advantageous for modelling of highly synchronized rhythms and firings, like slow-wave oscillations or epileptic seizures. Moreover, the sigmoidal subthreshold adaptation function allows one to model the dynamics of voltage dependent ion channels in more detail, while retaining the overall computational simplicity. The sigmoidal form of the adaptation function enriches the dynamics, allowing a wider repertoire of multi-stabilities.

However, our model has some limitations. First, the adaptation has the form of a non-inactivating current (such as $\mathrm{I}_{\mathrm{M}}$ potassium current) which limits the description of a class of inactivating ionic channels. It also includes only one type of subthreshold adaptation. In comparison to AdEx model, the computational cost of our model may be slightly higher due to the form of the adaptation variable, and more specifically the introduction of an exponential function. Also, as more parameters are introduced, more thorough dynamical studies and explorations of the parameter space are needed.

\section{Acknowledgement}

All simulations were done in Brian2 neural simulator and Python 3 programming language. The work was supported by CNRS and the European Community (Human Brain Project, H2020-785907). 


\section{Appendix}

\section{Bifurcations analysis}

\section{Rheobase current}

The derivative of function $S(V)$ is given by

$$
\begin{aligned}
S^{\prime}(V)= & g_{L}-g_{L} \exp \left(\frac{V-V_{T}}{\Delta_{T}}\right)+\frac{\bar{g}_{A}}{1+\exp \left(\frac{V_{A}-V}{\Delta_{A}}\right)} \\
& -\frac{1}{\Delta_{A}} \frac{\bar{g}_{A} \exp \left(\frac{V_{A}-V}{\Delta_{A}}\right)}{\left(1+\exp \left(\frac{V_{A}-V}{\Delta_{A}}\right)\right)^{2}}\left(E_{A}-V\right)
\end{aligned}
$$

The solution of the transcendental equation $S^{\prime}(V)=0$ gives a location $V^{*}$ of a maximum of $S(V)$ function, and consequently a rheobase current $I_{R}=S\left(V^{*}\right)$.

\section{Local linearized dynamics around equilibria}

The Jacobian of CAdEx system around equilibrium $i$, located at $\left(V^{(i)}, g_{A}^{(i)}\right)$, for an input current $I_{s}$ has a form:

$$
L_{i}\left(I_{s}\right)=\left(\begin{array}{cc}
-\frac{g_{L}}{C}+\frac{g_{L}}{C} \exp \left(\frac{V^{(i)}\left(I_{s}\right)-V_{T}}{\Delta_{T}}\right)-\frac{g_{A}^{(i)}\left(I_{s}\right)}{C} & \frac{E_{A}-V^{(i)}\left(I_{s}\right)}{C} \\
\frac{\bar{g}_{A}}{\tau_{A} \Delta_{A}} \frac{\exp \left(\frac{V_{A}-V^{(i)}\left(I_{s}\right)}{\Delta_{A}}\right)}{\left(1+\exp \left(\frac{V_{A}-V^{(i)}\left(I_{s}\right)}{\Delta_{A}}\right)\right)^{2}} & -\frac{1}{\tau_{A}}
\end{array}\right)
$$

The trace $\tau_{i}\left(I_{s}\right)$ and the determinant $\Delta_{i}\left(I_{s}\right)$ of Jacobian are as follows:

$$
\begin{aligned}
\tau_{i}\left(I_{s}\right) & =-\frac{g_{L}}{C}+\frac{g_{L}}{C} \exp \left(\frac{V^{(i)}\left(I_{s}\right)-V_{T}}{\Delta_{T}}\right)+\frac{g_{A}^{(i)}\left(I_{s}\right)}{C}-\frac{1}{\tau_{A}} \\
\Delta_{i}\left(I_{s}\right) & =\frac{g_{L}}{\tau_{A} C}-\frac{g_{L}}{\tau_{A} C} \exp \left(\frac{V^{(i)}\left(I_{s}\right)-V_{T}}{\Delta_{T}}\right)+\frac{g_{A}^{(i)}\left(I_{s}\right)}{\tau_{A} C} \\
& -\frac{\bar{g}_{A}\left(E_{A}-V^{(i)}\left(I_{s}\right)\right)}{\tau_{A} C \Delta_{A}} \frac{\exp \left(\frac{V_{A}-V^{(i)}\left(I_{s}\right)}{\Delta_{A}}\right)}{\left(1+\exp \left(\frac{V_{A}-V^{(i)}\left(I_{s}\right)}{\Delta_{A}}\right)\right)^{2}}
\end{aligned}
$$

From above equations:

$$
\Delta_{i}=-\frac{1}{\tau_{A}} \tau_{i}-\frac{1}{\tau_{A}^{2}}-\frac{\bar{g}_{A}\left(E_{A}-V^{(i)}\left(I_{s}\right)\right)}{\tau_{A} C \Delta_{A}} \frac{\exp \left(\frac{V_{A}-V^{(i)}\left(I_{s}\right)}{\Delta_{A}}\right)}{\left(1+\exp \left(\frac{V_{A}-V^{(i)}\left(I_{s}\right)}{\Delta_{A}}\right)\right)^{2}}
$$


The eigenvalues of Jacobian matrix at fixed points are then given by:

$$
\lambda_{12}=\frac{\tau \pm \sqrt{\tau^{2}-4 \Delta}}{2}
$$

If eigenvalues are complex, i.e. $\tau^{2}-4 \Delta<0$, then system oscillates around equilibrium. The imaginary part of an eigenvalue is equal to angular frequency of oscillation, i.e. $\operatorname{Im}(\lambda)=\omega=2 \pi \nu$. Consequently the frequency of oscillations is given by:

$$
\nu=\frac{1}{4 \pi} \sqrt{4 \Delta-\tau^{2}}
$$

\section{Conductance-based synapses}

To describe the behavior of the system receiving synaptic input, as in Section 4 concerning Multi-stability, we used a conductance-based model of synaptic inputs.

The synaptic input current to our model is given by the following equation:

$$
I_{s y n}=g_{E}\left(E_{E}-V\right)-g_{I}\left(E_{I}-V\right)
$$

Where $E_{E}=0 \mathrm{mV}$ is the reversal potential of excitatory synapses and $E_{I}=$ $-80 \mathrm{mV}$ is the reversal potential of inhibitory synapses. $g_{E}$ and $g_{I}$, are respectively excitatory and inhibitory conductances, which increase by quantity $Q_{E}=4 \mathrm{nS}$ and $Q_{I}=1.5 \mathrm{nS}$ on each incoming spikes. The increment of conductance is followed by exponential decrease according to equation:

$$
\frac{d g_{E / I}}{d t}=-\frac{g_{E / I}}{\tau_{s y n}}
$$

where $\tau_{\text {syn }}=5 \mathrm{~ms}$. The spikes trains are generated by Poissonian process with firing rate modulated by Ornstein-Ulhenbeck stochastic process (Fourcaud \& Brunel, 2002).

\section{References}

Brette, R., \& Gerstner, W. (2005). Adaptive exponential integrate-and-fire model as an effective description of neuronal activity. Journal of neurophysiology, 94 (5), 3637-3642.

Brown, D., \& Adams, P. (1980). Muscarinic suppression of a novel voltagesensitive $\mathrm{k}+$ current in a vertebrate neurone. Nature, 283(5748), 673.

Clopath, C., Jolivet, R., Rauch, A., Lüscher, H.-R., \& Gerstner, W. (2007). Predicting neuronal activity with simple models of the threshold type: Adaptive exponential integrate-and-fire model with two compartments. Neurocomputing, 70(10-12), 1668-1673. 
Connor, J. A., \& Stevens, C. F. (1971, Feb). Prediction of repetitive firing behaviour from voltage clamp data on an isolated neurone soma. J. Physiol. (Lond.), 213(1), 31-53.

Fitzhugh, R. (1961, Jul). Impulses and Physiological States in Theoretical Models of Nerve Membrane. Biophys. J., 1(6), 445-466.

Fourcaud, N., \& Brunel, N. (2002, Sep). Dynamics of the firing probability of noisy integrate-and-fire neurons. Neural Comput, 14 (9), 2057-2110.

Izhikevich, E. M. (2003). Simple model of spiking neurons. IEEE Transactions on neural networks, 14 (6), 1569-1572.

Krinskii, V. I., \& Kokoz, I. u. M. (1973). [Analysis of the equations of excitable membranes. I. Reduction of the Hodgkins-Huxley equations to a $2 \mathrm{~d}$ order system]. Biofizika, 18(3), 506-511.

McCormick, D. A., \& Contreras, D. (2001). On the cellular and network bases of epileptic seizures. Annu. Rev. Physiol., 63, 815-846.

Morris, C., \& Lecar, H. (1981, Jul). Voltage oscillations in the barnacle giant muscle fiber. Biophys. J., 35(1), 193-213.

Naud, R., Marcille, N., Clopath, C., \& Gerstner, W. (2008). Firing patterns in the adaptive exponential integrate-and-fire model. Biological cybernetics, 99(4-5), 335 .

Stimberg, M., Brette, R., \& Goodman, D. F. (2019, aug). Brian 2, an intuitive and efficient neural simulator. eLife, 8, e47314. doi: 10.7554/eLife.47314

Treves, A. (1993). Mean-field analysis of neuronal spike dynamics. Network: Computation in Neural Systems, 4(3), 259-284. doi: 10.1088/0954898X_4_3_002 